\title{
Multidisciplinary Design Optimization for the Plunger Mechanism of a Lubrication Pump \\ YixiangZhang ${ }^{1, a}$, Xifan Yao*1, b ${ }^{\text {, Cunji Zhang }}{ }^{1, c}$
}

\author{
${ }^{1}$ School of Mechanical \& Automative Engineering, South China University of Technology Guangzhou. \\ 510640, Guangdong province, China \\ a meyxzhang@163.com, ${ }^{*}$ mexfyao@scut.edu.cn, ${ }^{\mathrm{C}}$ zhang.cunji@mail.scut.edu.cn
} Keywords: Lubricationpump, Plunger mechanism, Multidisciplinary design optimization,
Approximate model, Isight.

\begin{abstract}
A ZJ-type high pressure lubrication pump is analyzed by multidisciplinary design optimization (MDO) method, where data are sampled via optimal Latin hypercube experiments, and the response surface models (RSMs) for the plunger piston and cylinder body of the pump are built.Lightweight design of the piston mechanism is conducted by using the improved collaborative optimization with a relaxation factor combined with sequential quadratic programming (SQP) algorithm. The results show that the approximate model accelerates the opimization speed greatly, the mass of optimized plunger mechanism is reduced by $8.03 \%$, and MDO can effectivelly deal with the coupling factors between the different components to reduce mass while the stress constraints and fatigue life are met.
\end{abstract}

\section{Introduction}

Traditional design methods tend to achieve a local optimum in a single discipline. With the increasing complexity of engineering problems, local optima can not meet the requirements to achieve global optima. Multidisciplinary design optimization(MDO) is such a global optimal method where a complex system is decomposed into different subsystems by using suitable decomposition optimization strategy, each subsystem using existing experience and knowledge for analysis and optimization, and considering the mutual restrictions and influence relations between various disciplines at the same time. MDO is a methodology essentially ${ }^{[1-3]}$, based on concurrent engineering theory, by fully exploring and using the coordination mechanism of interaction between the various disciplines to balance the conflicts in the system, andusing multi-disciplinary optimization method and optimization algorithms to seek optima of the system. MDO was first used in the field of aerospace $^{[4]}$, and has undertaken a wide range of applications in aircraft, automobiles, machine tools, robots, etc ${ }^{[5-6]}$. This paper takes a ZJ-type high pressure lubrication pump as an example, uses the approximate technology for the high computational cost target, and obtains approximate model with response surface method to replace the original design. Based on the integration of Solidworks and Ansys by Isight, the lightweight design of the piston mechanism is conducted by using improved collaborative optimization.

\section{The Construction of Approximate Model}

This study use Isight software to integrate and drive various sub-disciplines software to achieve optimization of the pump. Asanalysis software calculates costly, many iterationsare time-consuming.In order to improve the efficiency of optimization analysis, the response surface methodology (RSM)is used to obtain the approximate model. The RSM was first proposed by Box and Draper ${ }^{[7]}$, which combinesexperimental design with mathematical statistics. The RSM gets relatively precise approximate functional relationship in the local area through trials, and shows up through algebraic expression. The mathematical foundation of RSM is fully solid with good continuity and derivability, as it can remove value noise well and make the target response smoother.This approximate method compromises the fitting accuracy and efficiency well and is 
effective $^{[8]}$. The approximate models of sub-disciplines by using RSM reduce the time of the calling software. The solving time is shortened geometrically.

As for the studied case of the cylinder and piston,20 samples are collected by using optimal latin hypercube design (Opt LHD). This design method has a very good space-filling and balanced property, which enables all the test points to be distributed as uniformly as possible in the design space.Samples and corresponding response target results are shown in Table 1.

Tab.1 Tested resultsfor the cylinder based on Opt LHD

\begin{tabular}{cccccc}
\hline Test order & $\mathrm{d}_{1} / \mathrm{mm}$ & $\mathrm{d}_{2} / \mathrm{mm}$ & $\mathrm{d}_{3} / \mathrm{mm}$ & Maximum stress/Mpa & Volume $/ \mathrm{mm}^{3}$ \\
\hline 1 & 12.50 & 12.15 & 5.52 & 99.11 & 14016.59 \\
2 & 12.63 & 12.02 & 6.26 & 114.31 & 13828.01 \\
3 & 12.76 & 14.00 & 5.73 & 122.98 & 13437.58 \\
4 & 12.89 & 13.73 & 6.89 & 128.21 & 13175.77 \\
5 & 13.02 & 12.94 & 6.57 & 109.76 & 13378.61 \\
6 & 13.15 & 13.34 & 6.68 & 106.46 & 13203.25 \\
7 & 13.28 & 13.21 & 5.00 & 114.37 & 13535.44 \\
8 & 13.42 & 12.28 & 6.78 & 106.32 & 13293.20 \\
9 & 13.55 & 11.89 & 6.36 & 129.96 & 13416.88 \\
10 & 13.68 & 11.63 & 5.63 & 115.38 & 13571.83 \\
11 & 13.81 & 12.55 & 5.84 & 110.89 & 13270.57 \\
12 & 13.94 & 13.60 & 5.94 & 123.41 & 12944.32 \\
13 & 14.07 & 11.76 & 6.05 & 116.96 & 13266.28 \\
14 & 14.21 & 12.42 & 5.10 & 115.80 & 13257.67 \\
15 & 14.34 & 13.47 & 5.42 & 115.44 & 12893.66 \\
16 & 14.47 & 12.68 & 5.21 & 119.50 & 13050.26 \\
17 & 14.60 & 13.86 & 6.47 & 117.98 & 12438.47 \\
18 & 14.74 & 13.07 & 7.00 & 119.96 & 12429.35 \\
19 & 14.87 & 11.50 & 5.31 & 124.96 & 13081.26 \\
20 & 15.00 & 12.81 & 6.15 & 126.41 & 12556.47 \\
\hline
\end{tabular}

Similarly for the plunger, 45 samples and simulation experiments are carried out. Response targets are maximum stress, fatigue lifeand plunger volume.

The above-mentioned values of response targets are used to generate response surfaces, and parts of the results are shown in Figure1.
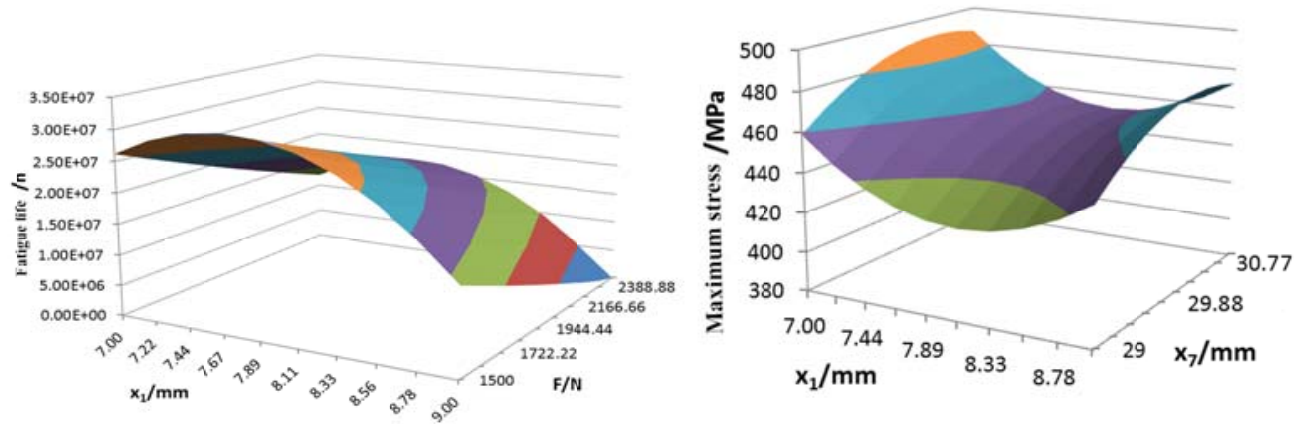

a) Response surface model of plunger piston's fatigue lifeb) Response surface model of plunger piston's stress
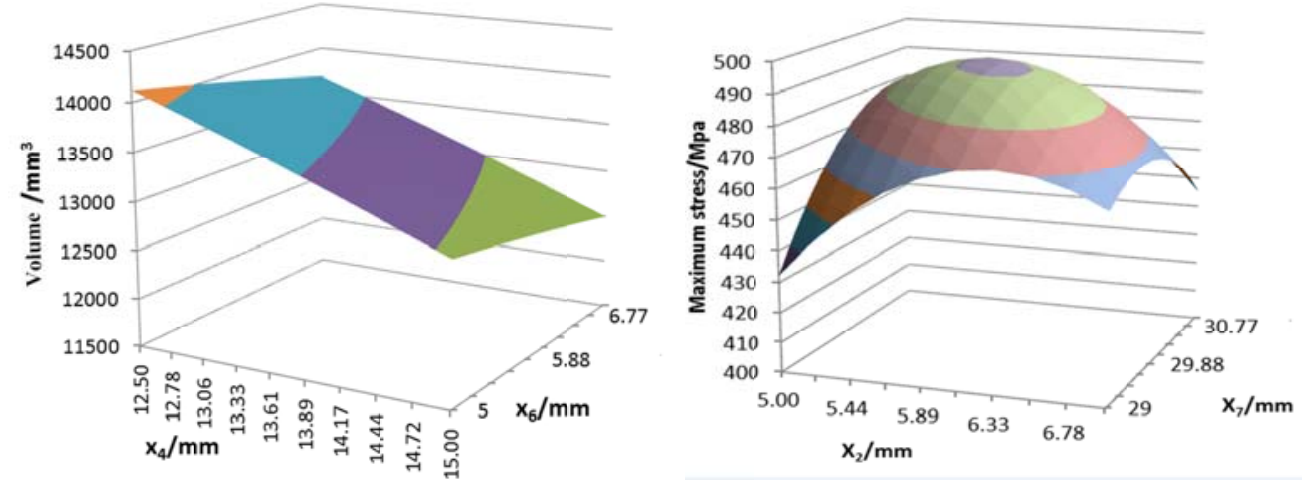

c)Response surface model of cylinder's volume d) Response surface model of plunger piston's stress

Fig.1 Parts of response surface models 


\section{The improved Collaborative Optimization}

Collaborative optimization(CO) approach is a kind of two level optimization method. An optimized object can be transformed into a system-level optimization and several subsystems optimization by the $\mathrm{CO}$ approach. Different sub-disciplinesare optimized separately in the subsystem-level. The linking variables and sharing variables in different sub-disciplines are coordinated by the consistency constraint conditions of the system-level. The advantage of the CO approach is that sub-disciplines can be optimized independently, so that the idea of concurrent engineering can be embodied. However, its convergence has not been rigorously proved, and it may be difficult to achieve convergence results in some cases. In this paper, an improved collaborative optimization approach based on the relaxed consistency constraint is adopted, where a relaxation factor cis added, and an inequality constraint is applied as consistency constraint instead of the strict equality constraint, so the convergence rate is speed up.Mathematical representation is as follows:

The system-level:

minimize
subject to $\quad J_{i}^{*}=\sum_{j=1}^{s_{i}}\left(z_{j}-x_{i j}^{*}\right)^{2}<\varepsilon, i=1,2, \ldots n(1)$

The ith subsystem:

subject to $\quad \mathrm{c}_{\mathrm{i}}\left(\mathrm{x}_{\mathrm{i}}\right) \leq 0(2)$

$$
\operatorname{minimize} \mathrm{J}_{\mathrm{i}}\left(\mathrm{x}_{\mathrm{i}}\right)=\sum_{\mathrm{j}=1}^{\mathrm{s}_{\mathrm{i}}}\left(\mathrm{x}_{\mathrm{ij}}-\mathrm{z}_{\mathrm{j}}^{*}\right)^{2}
$$

where $F(z)$ is system-level objective function, $J_{i}$ is system-level compatibility constraint, $c_{i}\left(x_{i}\right)$ is sub-discipline level constraint, $z_{j}$ is system-level design variable, $x_{i j}$ is the $j$ th design variable of the $i$ th subsystem, and $s_{i}$ is the number of design variables in the $i$ th subsystem.

\section{Lightweight Design of the Plunger Mechanism}

Collaborative optimization(CO) approach is applied in the plunger mechanism design. According to its physical components, a two-level optimization model is established: one system level and three subsystem levels(spool-spring optimization model, plunger optimization model, cylinder optimization model). The design goal is to minimize the total mass of the plunger mechanism. Each discipline must meet its size constraints and stress constraints, and the difference between optimized response values of sub-disciplines and allocated design variables of the system must be smallest tosatisfy consistency constraints. Consistency constraint of system level can coordinate the differences between disciplines.
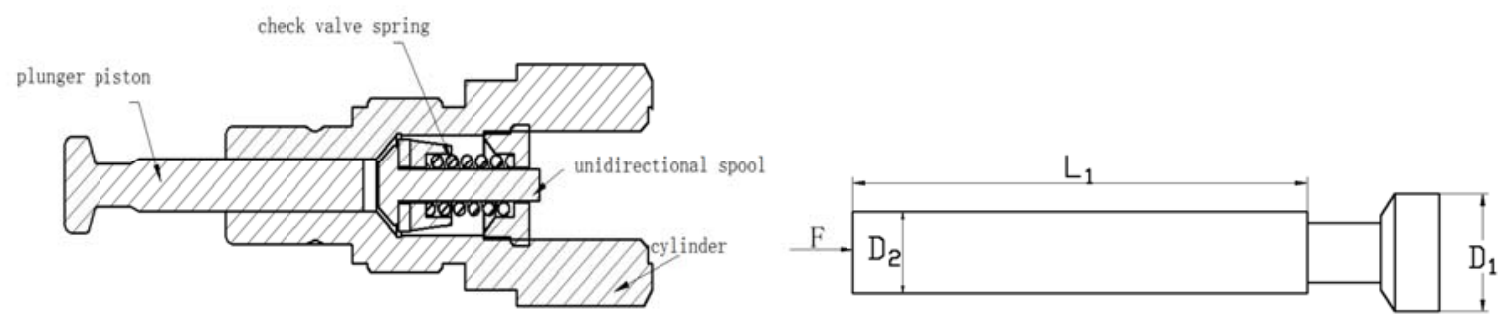

a) The plunger mechanismassembly b) The piston
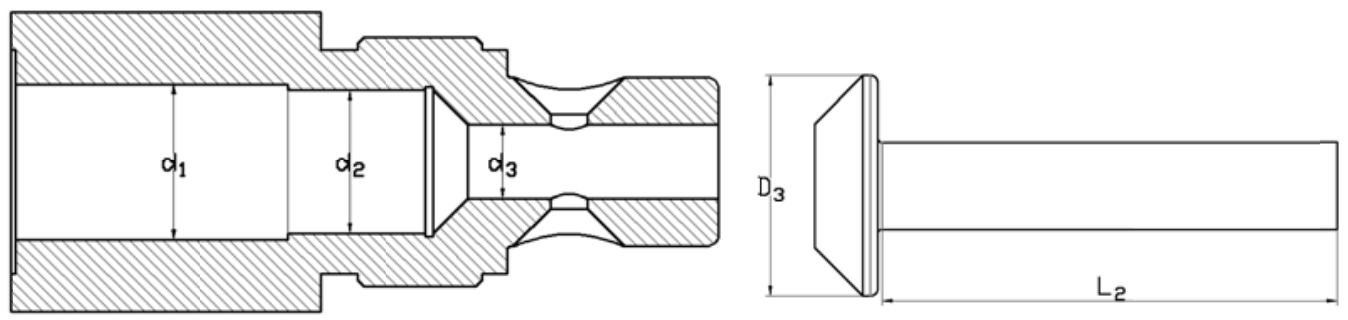

c) The cylinderd) The unidirectional spool

Fig.2 The plunger mechanism and its parts

The plunger mechanism and its parts are shown in Figure 2. Design variables include: the diameter of the plunger's large end face $D_{1}$ and small end face $D_{2}$, the length of the plunger $\operatorname{rod} L_{1}$, the length of 
unidirectional spool rod $L_{2}$ and diameter of end face $D_{3}$, three inner diameters of the cylinder $d_{1}$, $d_{2}$ and $d_{3}$, and the wire diameter of check valve spring $f$. Grease pressure $P$, the plunger rod force $F$ and the spring stiffness coefficient Kare system linking variables.

$$
\begin{array}{r}
\mathrm{X}=\left(D_{1}, D_{2}, D_{3}, d_{1}, d_{2}, d_{3}, L_{1}, L_{2}, f\right)=\left(x_{1}, x_{2}, x_{3}, x_{4}, x_{5}, x_{6}, x_{7}, x_{8}, x_{9}\right) \\
\mathrm{Y}=(P, F, K)=\left(y_{1}, y_{2}, y_{3}\right)(3)
\end{array}
$$

Geometric constraints of design variables are shown in Table 2.The subsystem variables $x_{2}$ and $x_{6}$ arethe shared design variables in system level.Sequential quadratic programming (SQP) is used as a numerical optimization algorithm, where convergence precision is set to be $10^{-6}$, relative step 0.001 , and the minimum absolute step $10^{-4}$. Integrated optimization framework is shown in Figure 3.The system level converged after 253 iterations, and the results are shown in Figure 4.

Tab.2 Initial value and geometric constraints of design variables

\begin{tabular}{cccc}
\hline Design variable/mm & Initial value & Lower limit & Upper limit \\
\hline$x_{1}$ & 8.00 & 7.00 & 9.00 \\
$x_{2}$ & 5.50 & 5.00 & 7.00 \\
$x_{3}$ & 9.10 & 8.00 & 10.00 \\
$x_{4}$ & 12.50 & 11.50 & 15.00 \\
$x_{5}$ & 11.50 & 10.00 & 14.00 \\
$x_{6}$ & 5.50 & 5.00 & 7.00 \\
$x_{7}$ & 31.00 & 29.00 & 32.00 \\
$x_{8}$ & 19.00 & 15.00 & 22.00 \\
$x_{9}$ & 1.50 & 1.00 & 1.90 \\
\hline
\end{tabular}

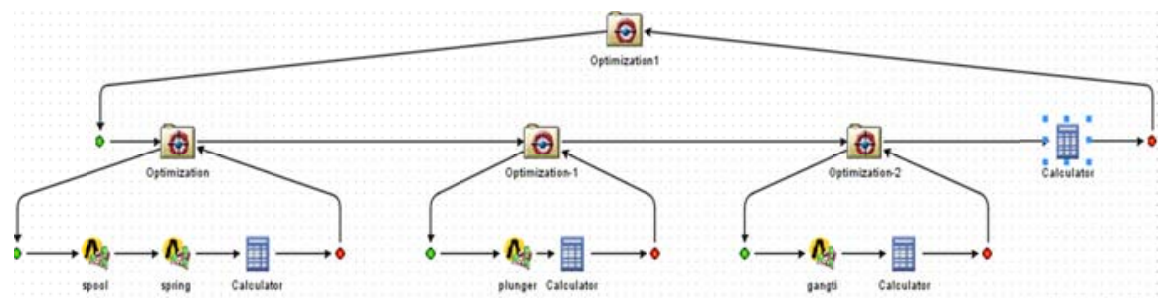

Fig.3 Integratedoptimization framework for the plunger mechanism

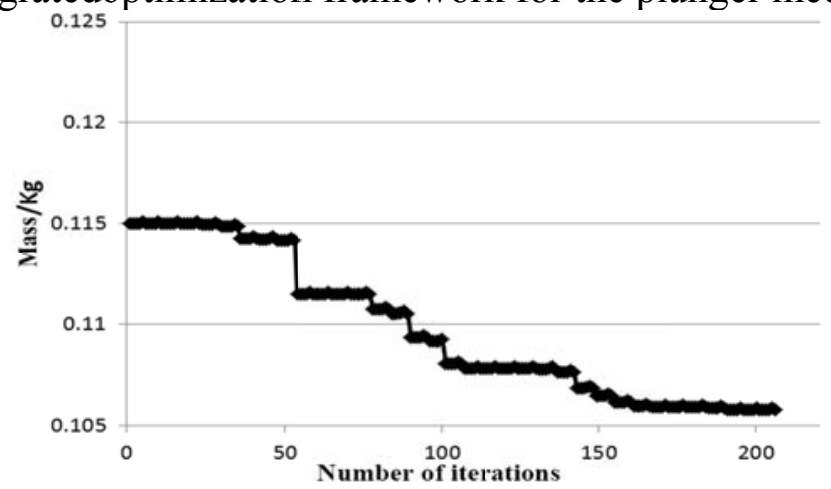

Fig.4 Optimization results forPlunger mechanism's mass

Tab.3 Optimization variables and results of plunger mechanism

\begin{tabular}{ccc}
\hline Design variable $/ \mathrm{mm}$ & Initial value & Optimization result \\
\hline $\boldsymbol{x}_{\mathbf{1}}$ & 8.00 & 7.00 \\
$\boldsymbol{x}_{\mathbf{2}}$ & 5.50 & 5.21 \\
$\boldsymbol{x}_{\mathbf{3}}$ & 9.10 & 9.52 \\
$\boldsymbol{x}_{\mathbf{4}}$ & 12.50 & 15.00 \\
$\boldsymbol{x}_{\mathbf{5}}$ & 11.50 & 14.00 \\
$\boldsymbol{x}_{\mathbf{6}}$ & 5.50 & 5.08 \\
$\boldsymbol{x}_{\mathbf{7}}$ & 31.00 & 29.00 \\
$\boldsymbol{x}_{\mathbf{8}}$ & 19.00 & 15.28 \\
$\boldsymbol{x}_{\mathbf{9}}$ & 1.50 & 1.06 \\
\hline
\end{tabular}

Optimization results of design variable are shown in Table 3.The mass of plunger mechanism decreased from $0.1150 \mathrm{~kg}$ to $0.1058 \mathrm{~kg}$ after optimization, and there is a reduction of $8.03 \%$. 


\section{Conclusion}

The plunger mechanism of lubrication pump is used as a case of study, and response surface approximation model is adopted to improve the efficiency of optimization. After the optimization of the plunger mechanism, the overall mass is reduced by $8.03 \%$. The purpose of optimization is achieved. In this study, the MDO method is first used in the plunger mechanism of lubrication pump, which can provide a new approach for the design of other plunger mechanisms.

\section{Acknowledgments}

The project was supported by the National Natural Science Foundation of China under grant No.51175187, and the Science \& Technology Foundation of Guangdong Province under grant Nos. 2014A020223003, and 2015A020220004.

\section{References}

[1] I.P. Sobieski, I.M.Kroo, Collaborative optimization using responsesurface estimation, AIAA Journal, vol. 38, no. 10,pp.1931-1938, 2000.

[2] S. Kodiyalarn, J.S.Sobieski, Bi-level integrated system synthesis with response surface, AIAA Journal,vol. 38, no. 8,pp.1479-1485, 2000.

[3] J. Sobieszczanski-Sobieski. Sensitivity of complex, internally coupled systems. AIAA Journal,vol. 28, no. 1, pp. 153-160,1990.

[4] J. Sobieszczanski-Sobieski, Optimization by decomposition: a step from hierarchic to Non-hierarchic system, Hampton VA: NASA Langley Research Center, 1988.

[5] A.D. Yin, X.Y. Dong, B.Z. Zhang, et al,Multi-objective optimization of control parameters of range-extended electric vehicle based on Isight, Journal of Hefei University of Technology, vol. 38, no. 8, pp. 289-294,2015.

[6] B. Li, Multidisciplinary design co-optimization for mechatronics product with 6-dof manipulator as an example, Guangzhou: South China University of Technology, 2014.

[7] G.E.P. Box, K.B. Wilson, On the experimental attainment of optimum conditions, Journal of the Royal Statistical Society,vol. 13, no. 1,pp. 1-45, 1992.

[8] C. Bucher, T. Most, A comparison of approximate response functions in structural reliability analysis, Probabilistic Engineering Mechanics,vol. 23, no. 2,pp. 154-163, 2008. 